\title{
Arteriovenous Malformations: Clinical Aspects and Surgical Results
}

\author{
Momar Sokhna Diop*, Papa Adama Dieng, Magaye Gaye, Ndeye Fatou Sow, Amadou Gabriel Ciss, \\ Papa Salmane Ba, Papa Amath Diagne, Souleymane Diatta, Assane N'diaye, Mouhamadou N'diaye \\ Department of Cardiovascular and Thoracic Surgery, Cheikh Anta Diop University, Dakar, Senegal
}

\section{Email address:}

momarsokhna08@yahoo.fr (M. S. Diop),padiengsala@yahoo.fr (P. A. Dieng), cissgaby@yahoo.fr (A. G. Ciss), mansalb@yahoo.fr (P. S. Ba), mgaye99@yahoo.fr (M. Gaye), zenefes@gmail.com (N. F. Sow),

diagnepapaamathdiagne@gmail.com (P. A. Diagne), soul.diatta@yahoo.fr (S. Diatta), lazanat@yahoo.fr (A. N'diaye),

mondiaye@orange.sn (M. N'diaye)

${ }^{*}$ Corresponding author

\section{To cite this article:}

Momar Sokhna Diop, Papa Adama Dieng, Magaye Gaye, Ndeye Fatou Sow, Amadou Gabriel Ciss, Papa Salmane Ba, Papa Amath Diagne, Souleymane Diatta, Assane N'diaye, Mouhamadou N'diaye. Arteriovenous Malformations: Clinical Aspects and Surgical Results. International Journal of Cardiovascular and Thoracic Surgery. Vol. 4, No. 3, 2018, pp. 20-25. doi: 10.11648/j.ijcts.20180403.11

Received: May 12, 2018; Accepted: June 4, 2018; Published: June 28, 2018

\begin{abstract}
Arteriovenous malformations (AVM) are vascular malformations, broadband, formed of arterial and venous dysmorphic vessels interconnected directly without transition in a capillary bed. Arteriovenous malformation surgery is particularly demanding owing to the need to control bleeding. This is probably one of the most critical moments of arteriovenous malformation surgery. The purpose of this study is to analyzed the clinical aspects and evaluate the results of surgery in the treatment of these AVMs. This is a retrospective and descriptive study that took place in Dakar over a period from January 2004 to December 2017 on patients operated for arteriovenous malformations. The total number of our series was 11 cases. These AVMs represented $32.35 \%$ of all tumors and vascular malformations operated during this period. The mean age at surgery was 25 years old [1 year-56 years old]. A male predominance was noted with 7 male to 4 female (sex ratio 1.75). The average time of consultation was 8.3 years [ 1 months-30 months]. The main reason for consultation was the appearance of a mass in 11 cas. We noted a notion of traumatism or recent surgery were done in $4 / 11$ before the onset of symptoms. The location of the lesions was at the head or the face ( 5 cases), neck ( 1 case), the upper member ( 2 cases), the lower member ( 3 cases). No multiple location were noted. After physical examination, two patients were classified Schobinger stage 1, stage 2 in 7 cases, 2 cases in stage 3. No patient was classified stage 4 . The vascular Doppler ultrasound was performed in 10 cases $(91 \%)$ and allowed to confirm the diagnosis in 10 cases. The CT angiography was performed in 8 cases (73\%). It elicited the feeding artery and draining veins, the number of nidus and topography. We found 6 truncal AVM and 5 extratruncal including 4 limited. A first embolization was performed in 2 cases (18.2\%). A one-stage surgery was performed in 7 cases and two times in 4 cases. The average hospital stay was 15 days [ 3 days-60 days]. The average healing time was 18 days [15 days-30 days]. Operative mortality as early mortality was zero. Late mortality was zero. The average follow-up time was 50.3 months [2 months-96 months]. In recent years, the multidisciplinary approach of tumors and vascular malformations has made important advances in the delineation of nosological frameworks and in the understanding of the natural history and structure of these complex lesions. This is why the treatment of these AVMs requires a multidisciplinary consultation exchange between vascular surgeons, plastic surgeons, interventional radiologists and anesthetists.
\end{abstract}

Keywords: Arteriovenous Malformation, Surgery, Senegal

\section{Introduction}

Vascular abnormalities are divided into two groups: tumors and vascular malformations. In these vascular malformations, we find the arteriovenous malformations (AVMs) which are high-flow vascular malformations of directly interconnected dysmorphic arterial and venous vessels without the transition of a capillary bed 
[1]. Frequently discrete at the beginning stage, they can pose differential diagnosis problems with other less severe vascular abnormalities. AVMs acquire, over time, an increasingly aggressive evolutionary profile, which causes significant therapeutic difficulties [2]. In most cases, the AVMs arises from the intracranial area, but occasionally originates from extracranial vessels [3]. Their management is often problematic, requiring in a quasi-systematic way often the assistance of a multidisciplinary consultation [2]. This study aims to analyze the clinical aspects and to evaluate the results of the surgery in the treatment of these arteriovenous malformations.

\section{Materials and Methods}

This is a retrospective descriptive study that took place in Dakar over a period from January 2004 to December 2017, focusing on patients who underwent surgery for arteriovenous malformations. The data was collected from records saved at the archives level of the Cardiovascular Surgery Clinic. These patients were operated in Dakar for arteriovenous malformations. A survey card was prepared for each patient for this study. All patients undergoing arteriovenous malformation with an archival record at the Cardiovascular Surgery Clinic at the Fann National University Hospital Center were included in the study. All patients whose records were incomplete with respect to the endpoints studied and patients with AVMs monitored or medically treated were excluded from the study. The collected data are analyzed with the Excel software.

\section{Results}

The total number of our series was 11 cases. These arteriovenous malformations accounted for $32.35 \%$ of all tumors and vascular malformations performed during this period. The mean age at the time of surgery was 25 years [ 1 to 56 years]. A male predominance was noted with 7 male for 4 female (sex ratio 1.75). A notion of family consanguinity was found in 2 cases / 11 . The average consultation period was 8.3 years [ 1 month-30 months]. The main reason for consultation was the appearance of a mass in 11 cases associated with ulceration ( 2 cases), intermittent claudication ( 2 cases), localized hyperthermia (1 case). A recent trauma or surgery was reported in 4 cases / 11 before the onset of symptoms. The lesions were located at the head or face $(5$ cases), neck (1 case), upper limb (2 cases), lower limb (3 cases). No multiple locations were noted. On clinical examination 7 cases (64\%) of these swellings had vascular characteristics. A collateral venous circulation was present in 5 cases $(45 \%)$. No deformity or nervous deficit was found. At the end of the physical examination, 2 patients were classified as stage 1 of Schobinger, 7 in stage 2, 2 in stage 3. No patient was classified as stage 4. Vascular echocardiography was performed in 10 cases $(91 \%)$ and confirmed the diagnosis in 10 cases. CT angiography was performed in 8 cases $(73 \%)$. It made it possible to specify the feeder artery and drainage veins, the number of nidus and their topography.

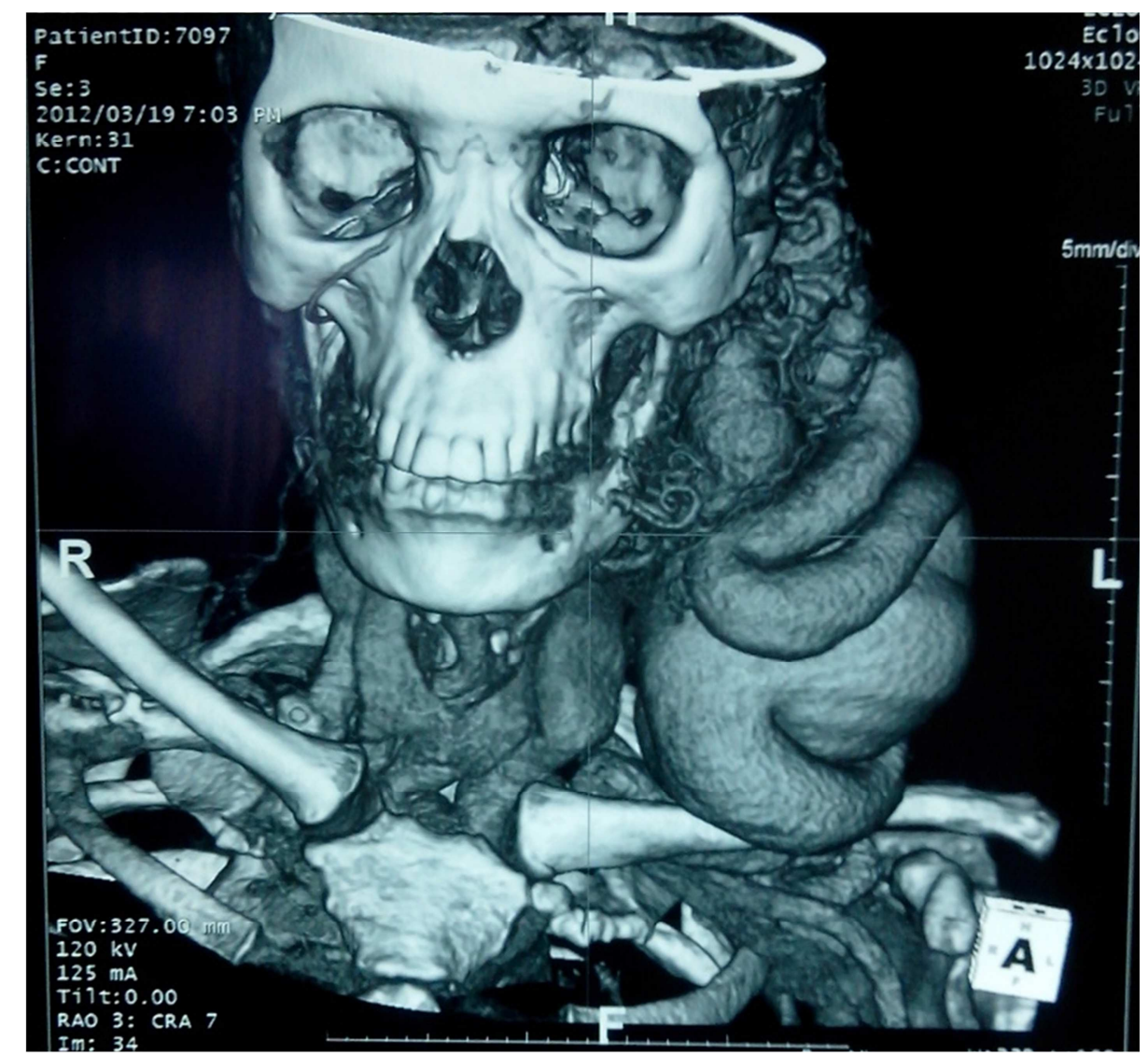

Figure 1. Show a CT angiography view of a neck's arteriovenous malformation. 
In a patient, the $\mathrm{CT}$ angiography found images of erosion of the fibula associated with a right surgical arteriovenous malformation. Nuclear magnetic resonance imaging (MRI) was performed in 2 cases $(18.2 \%)$. The arteriography was performed in 3 cases and it was possible to find in a case where the CT angiography was non-contributing an AVM fed mainly by the posterior tibial artery with anastomoses with the peroneal. In the other case where the CT angiography was not performed, there was an AVM located at the anterior part of the right thigh fed by a branch of the superficial femoral artery and draining into an accessory saphenous vein. The surgical indication was based on an esthetic application in 7

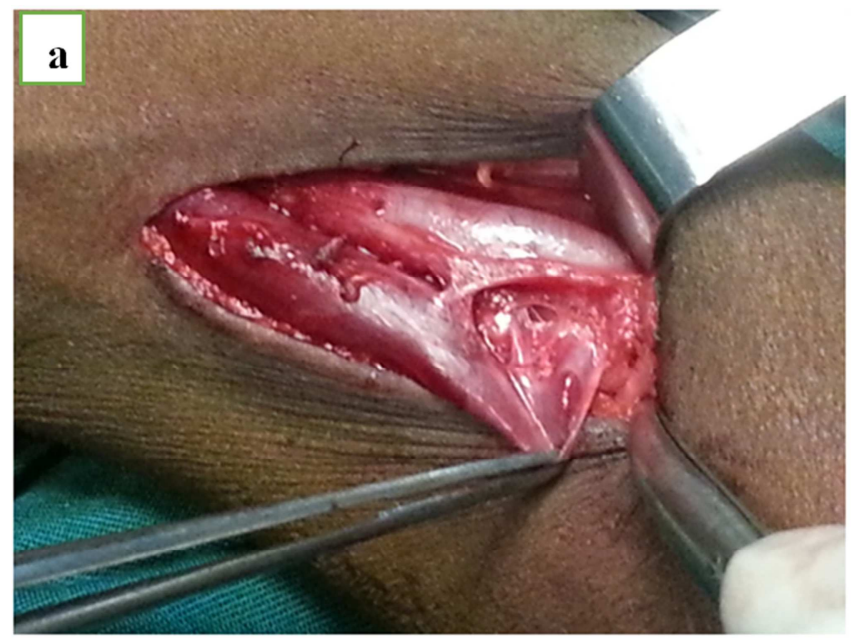

cases / 11, intermittent claudication in 2 cases / 11 and ulcerations in 2 cases / 11. There were 6 truncular AVM and 5 extratruncular, including 4 limited. Initial embolization was performed in 2 cases $(18.2 \%)$ using curaspon. Surgery was performed in all cases. One-time surgery was performed in 7 cases and in 2 cases in 4 cases. The surgical gestures consisted of a ligature of the alimentary artery in 8 cases of which 2 were associated with a ligature of the drainage vein. Secondarily, total resection was performed in 4 cases / 11. A total resection of the forearm in one time was performed for a forearm and hand AVM.

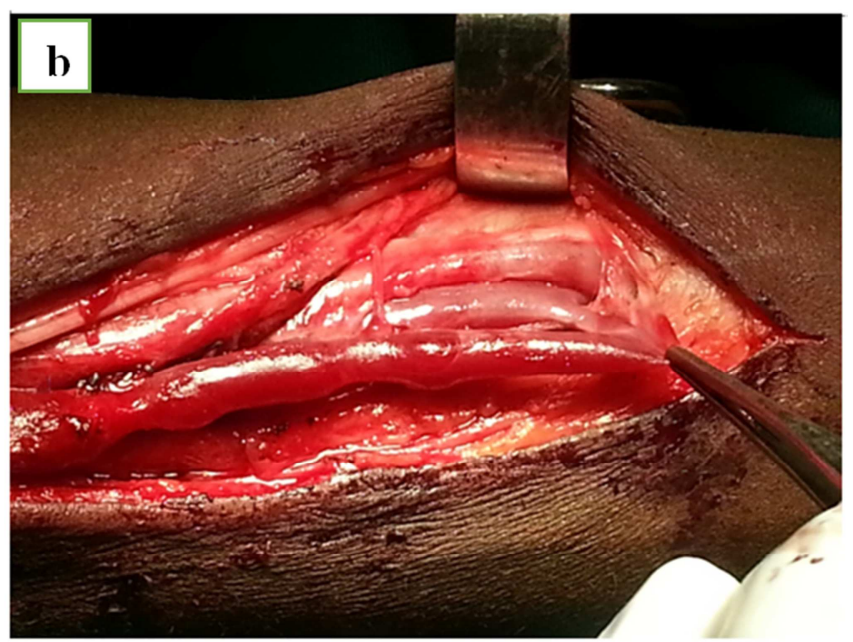

Figure 2. a) Show an operative view of an upper limb arteriovenous malformation; b) Show an operative view with clips on an AVM of the upper limb.

The mean duration of the intervention was 130 minutes [60 minutes-300 minutes]. The average duration of hospitalization was 15 days [3 days-60 days]. The average healing time was 18 days [15 days-30 days]. Complementary gestures were required in 5 patients ( 2 cases, embolization 2 cases, multiple sclerosis 3 cases). Complications were observed in 5 patients $(45.4 \%)$ with hemodynamic, thromboembolic, metabolic, neurological and ischemic complications. Both operative mortality and early mortality were nil. Late mortality was nil. Follow-up was performed in 10 patients. The mean duration of follow-up was 50.3 months [2 months-96 months]. The progression was favorable in 6 patients / 10 with an eutrophic surgical scar. No clinical symptoms were individualized. The vascular echocardiography performed in 5 patients did not obviate anomalies. No recurrence was observed in these patients. A patient who had undergone a treatment for deep right truncal AVM with a resection was not performed, showed signs of venous insufficiency at 18 months of follow-up. Treatment with compression stockings associated with veinotonics was initiated. Revised at 61 months postoperatively, it still showed non-systemic varicose dilatations in the right leg with a bulky calf without thrill or breath and there was no Doppler flow. The venous ulcer was healed. Three out of 10 patients, or $30 \%$, had recurrences.

\section{Commentary}

In the literature, AVMs represent only $10-15 \%$ of vascular malformations, but the true incidence of vascular malformations is underestimated $[4,5]$. Some even think that the previous classifications have created nosological confusions [6]. In this series these AVMs represent 32.35\% of all tumors and vascular malformations operated during this period. In the Diarra series [7], these AVMs represent $15 \%$ of all the vascular malformations studied. In our study, there is a male predominance with an average age at diagnosis of 25 years. Kim [5] finds an average age of 28 years with a female predominance (sex ratio of 0.7). Guero [8] says there is no gender predominance. In Saeed Kilani's study the average age was 36.5 years [2-72 years] [9]. The diagnosis made in adolescence could be explained by the existence of triggering factors such as hormonal changes occurring during this period. Other factors such as pregnancy, infections, trauma, surgical procedures are also implicated [4]. The age of development is variable according to Enjorlas and al. [10] (40\% are visible at birth, $35 \%$ appear in childhood and $25 \%$ appear later in adults). Liu and al. [11] finds that children who have stage 1 Schobinger AVMs presented $43.8 \%$ risk of aggravation in adolescence and $82.6 \%$ in adulthood. The average consultation time in the Guillet study [12] is 10.4 
years. This was close to the diagnostic time of patients in this serie. In the study by Osuga and al. [13], pain and swelling are the predominant symptoms found in $17 / 25$ and $24 / 25$, respectively. The main localizations of the AVMs are head and nose in $40 \%$, limbs in $40 \%$ and trunk in $20 \%$ [14]. This was the case in this series. Compared to the Guillet series [12], the majority of our patients are classified in stage 2 of the Schobinger classification. In this series the vascular echodoppler was able to make the diagnosis in 10 cases / 11 and the CT angiography to specify the feeder artery, the drainage veins and the topography of the nidus. Johnson and al. [15] advocates starting with magnetic resonnance imanging (MRI). The criticism he brought back for echoing was that he could not examine the deep lesions and those located near the bone structures or containing air. In this series, only two patients had MRI. It is an examination that is not always accessible and that the diagnosis is made on the basis of the vascular echodoppler supplemented with the CT angiography. In this series, in a patient with a right surgical AVMs, the CT angiography found images of fibula erosions. In a study conducted by Enjorlas, he noted 3 cases of bone lysis, very painful and at risk of spontaneous fractures (stage 3 of Schobinger) [10]. Laurian [16] states that deep tissue complications are represented by bone complications in contact with a high-throughput AVMs. The venous dilatations downstream of the shunt destroy the cortical bone and invade the medulla with corollary bone fragility. The indication of surgery on an AVM of a limb remains exceptional; it corresponds to particular lesional profiles [16]. In this series, the dominant indication is the cosmetic attack although in 4 cases it was an intermittent claudication or ulcerations. Kansy and al. says the timing of surgery remains a point of discussion. In their cohort, all patients experienced esthetic impairment, varying from asymmetry (swelling) to more severe disfigurement. Early treatment in these patients would require less invasive procedures, with a higher likelihood of therapy success. In the craniofacial region, the timing of surgical resection remains a decision based on individual patients, outweighing the potential risks and consequences of treatment against current complaints and the expected course without intervention [17]. In the study by Lee and al. [6], the majority of patients treated have extratruncular lesions. In this series, we find the truncal and extratruncular involvement in the same proportions. The therapeutic strategy adopted before an AVM diagnostic has always and continues to pose problems. Lee [6] adopted the strategy of preoperative embolism / sclerotherapy followed by a complete resection of the AVMs and this had reduced its morbidity compared to patients who had an initial excision. And in his study, surgical excision was done within 2 to 4 weeks after embolism / sclerotherapy. In the Kim series [5], of the 21 patients who underwent surgical treatment, 20 of them had preoperative embolo / sclerotherapy sessions. In the literature, the majority of surgeons recommend preoperative embolism / sclerotherapy [18]. In Kansy's study [17], he relied on the Heidelberg treatment algorithm for high-flow AVMs (see figure 3).

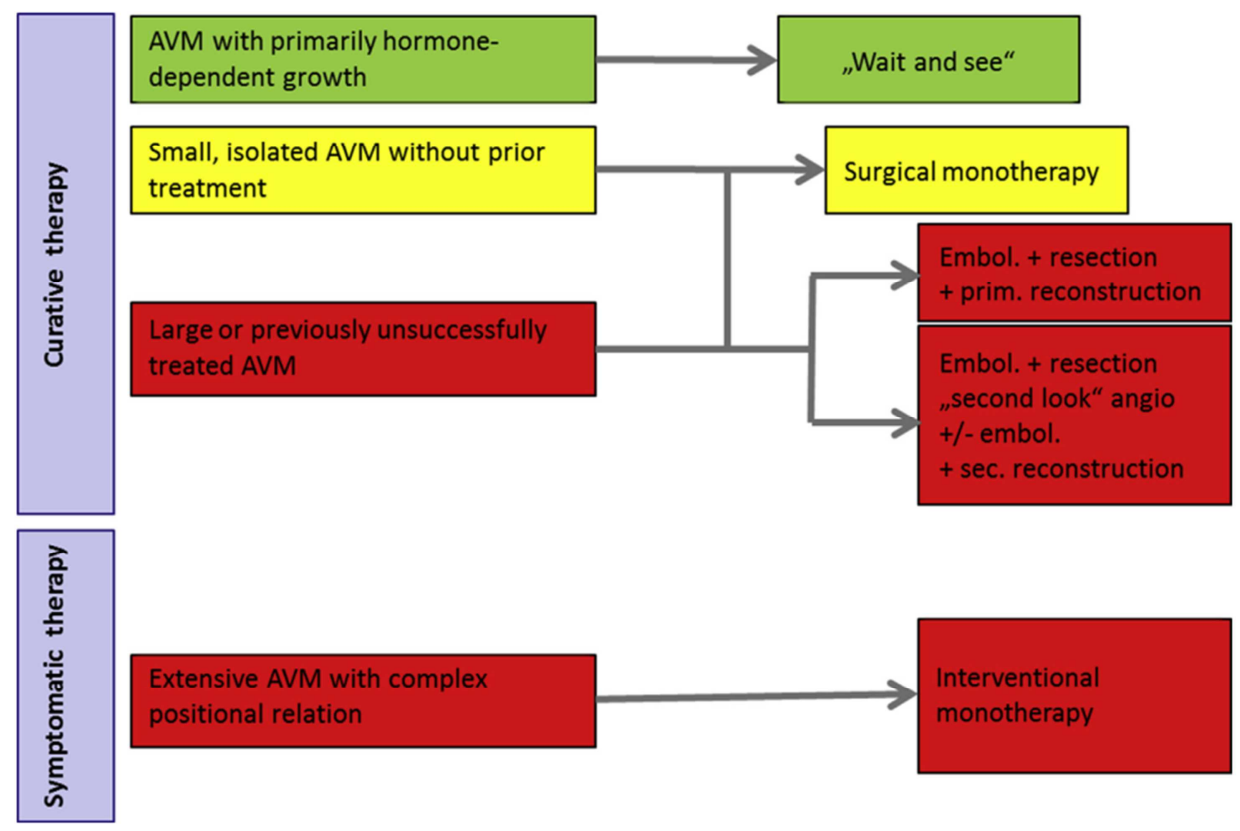

Figure 3. Show the Heidelberg treatment algorithm for high flow AVMS.

In this series, only two patients had first embolization. The main attitude consisted in ligation of the alimentary artery (8 cases). In 2 cases, a ligature of the drainage vein is associated. This attitude is often questioned. The argument that he reports is that by linking the feeder artery without removing the nidus, the other sleeping collateral of the AVMs grow rapidly and make the AVMs even more important [5]. And the other reason is, after the ligature of the alimentary artery, it is impossible to carry out a complementary embolo / sclerotherapy. And it is this very reason that has led Riles [19] to reconstruct a bound external carotid artery using either the saphenous vein or a polytetrafluoroethylene 
(PTFE) prosthesis in order to be able to carry out secondary embolization of the AVMs. In this study, of these 8 patients who benefited from a feeder ligation, 4 of them had a complete resection of the AVMs. Only one patient received total resection right away in one time for a forearm and hand AVM. De Miguel [4] argues that embolization should be performed 24 to 72 hours before surgery to reduce blood loss. However, some radiologists perform arterial and venous embolization at the same time and this is the only treatment and the results are the same as surgery especially in complex locations such as the head or nose. However, this technique requires several interventions. In one patient, peripheral facial paralysis was observed after submandibular AVM surgery with good clinical course under corticosteroid therapy and physiotherapy. Chen [20] finds in his series a case of peripheral facial paralysis which was regressive spontaneously in two weeks. De Miguel [4] systematically undergoes corticosteroid therapy in postoperative patients. Mortality (operative, early and late) is nil in our series as in most surgical series [5, 6, 20, 21]. The mean follow-up in our series is 50.3 months [2 months-96 months] with a favorable outcome in $60 \%$ of patients. In the Guillet series [12], followup is 57.6 months. He observed $31 \%$ complete remissions, $31 \%$ partial remissions, $15 \%$ recurrences after improvement and $23 \%$ treatment failures. In this series, there were signs of venous insufficiency in a patient at 18 months of follow-up. This accounts for the difficulty of managing infiltrative AVMs. Lee [6], in his series, finds 32 patients who have surgically inaccessible AVM and have benefited from embolism / isolated sclerotherapy with a success rate of $78 \%$. In the study of Saeed Kilani 12 out of 19 patients have obtained complete devascularization with embolization and surgical excision was performed in 9 patients [9]. In the $\mathrm{Su}$ study where he used ethanol to perform embolization treatment of AVM of the face, 56 of the 66 patients were cured with $100 \%$ of AVM devascularization, with the remaining 10 having partial remission with various degrees of AVM devascularization [22]. Embolism / sclerotherapy is a particularly interesting therapeutic modality for surgically inaccessible lesions [23-24]. The exclusion of the main arterial pedicle in our case has allowed us to have an inert AVM. The only consequence is the presence of signs of venous insufficiency and cosmetic impairment in a patient controlled by elastic restraint. In our study, 3 patients, or $30 \%$, experienced recurrence, and we note that these recurrences occurred in patients who received nourishing pedicle ligation without resection of the nidus for AVM located mainly on the head and face. In Kansy's series, patients were followed up for a mean of 26,8 months [range 12-60]. Combined treatment $(n=9)$ was free of recurrence in all cases $(100 \%)$. Surgical monotherapy $(n=2)$ was also successful in both cases. Non-curative interventional monotherapy led to a significant decrease in symptoms and did not have to be repeated [17]. Surgeons divide the topography of extracranial AVM into four sites (head and nose, viscera, upper limbs and lower limbs). Each site has its specific characteristics and when the surgery is indicated, it will be necessary to evaluate the benefits and the risks. The head and nose are considered critical areas and that removal with healthy margins is extremely difficult [4]. This is what led us to do a ligation of the pedicle feeder in these patients even if this attitude remains controversial. Juskat [25] performed an embolism / sclerotherapy for a cervical AVM with a favorable evolution. This was the case for 3 of our patients in whom only a ligation of the pedicles was carried out. In the Kim's study, twenty patients underwent therapeutic embolization. Of these, the AVMs improved in 3, but became aggravated in 17. Of the latter 17, 10 underwent AVM mass excision or auricular resection [26]. This shows the difficulty of managing cervical AVMs.

\section{Conclusion}

In recent years, the multidisciplinary approach of tumors and vascular malformations has made important advances in the delineation of nosological frameworks and in the understanding of the natural history and structure of these complex lesions. This is why the treatment of these AVM requires a multidisciplinary consultation exchange between vascular surgeons, plastic surgeons, interventional radiologists and anesthetists.

\section{References}

[1] Garzon MC, Huang JT, Enjorlas O, Frieden IJ. Vascular malformations. Part I. J Am Acad Dermatol 2007; 56: 353-70.

[2] Naouri M, Lorette G, Barbier C, Zakine G, Herbreteau D. Malformations artérioveineuses. Presse Med 2010; 39: 465470 .

[3] Raghu M., De R., Higgins N., Axon P. Spontaneous arteriovenous malformation of the external auditory meatus. J Laryngol Otol. 2004; 118: 912-3.

[4] De Miguel R, Lopez-Gutierrez JC, Boixeda P. Arteriovenous Malformations: A Diagnostic and therapeutic challenge. Actas Dermosifiliogr 2014; 105 (4): 347-358.

[5] Kim JY, Kim DI, Do YS, Lee BB, Kim YW, Shin SW et al. Surgical treatment for congenital arteriovenous malformation: 10 years experience. Euro J. Vasc Endovasc. Surg 2006; 32: 101-106.

[6] Lee BB, Do YS, Yakes W, Kim DI, Mattassi R, Hyon WS. Management of artriovenous malformations: A multidisciplinary approach. Journal of vascular surgery 2004; 39: 590-600.

[7] Diarra O, Ba M, Ndiaye A, Ciss G, Dia A, Ndiaye M. Anomalies vasculaires congénitales en chirurgie vasculaire en milieu africain: à propos de 28 cas colligés au CHU de Dakar. Journal des maladies vasculaires 2003; 28 (1): 24-29.

[8] Guero S. Tumeurs et malformations vasculaires des members. Chirurgie de la main 2007; 26: 278-287.

[9] Saeed Kilani M., Lepennec V., Petit P., Magalon D., Casanova D., Bartoli J-M., Vidal V. Embolization of peripheral highflow arteriovenous malformation with Onyx. Diagnostic and Interventional Imaging (2017) 98, 217-226. 
[10] Enjorlas O, Logeart I, Gelbert F, Lemarchand-Venencie F, Reizine D, Guichard JP et al. Malformations artérioveineuses: étude de 200 cas. Ann Dermatol Venereol 2000; 127 (1): 1722.

[11] Liu AS, Mulliken JB, Zurakowski D, Fishman SJ, Greene AK. Extracranial arteriovenous malformations: natural progression and recurrence after treatment. Plast Reconstr Surg 2010; 125: $1185-94$.

[12] Guillet A, Maruani A, Berton M, Herbreteau P, Perret C, Connault $\mathrm{J}$ et al. Signes cliniques précoces et pronostic à long terme des malformations artérioveineuses cutanées distales de membre: étude rétrospective multicentrique de 19 patients. Annales de dermatologie et vénéréologie 2014; 141 (12): 299300 .

[13] Osuga K, Hori S, Kitayoshi H, Khankan AA, Okada A, Sugiura $\mathrm{T}$ et al. Embolization of high flow arteriovenous malformations: experience with use of superabsorbent polymer microspheres. Journal of vascular interventional radiology 2002; 13: 1125-1133.

[14] Hyodoh H, Hori M, Akiba H, Tamakawa M, Hyodoh K, Hareyama M. Peripheral vascular malformations: imaging, treatment approaches and therapeutic issues. Radiographics 2005; 25: 159-171.

[15] Johnson JB, Cogswell PM, Mcknsick MA, Binkovitz LA, Riederer SJ, Young PM. Pretreatment imaging of peripheral vascular malformations. Journal of vascular diagnostics 2014; 2: $121-126$.

[16] Laurian C, Fransceschi C, Herbreteau D, Enjorlas O. Traitement chirurgical des malformations vasculaires des membres. EMC Chirurgie. 2004; 1: 100-124.

[17] Kansy K., Bodem J., Engel M., Freudlsperger C., Mohlenbruch M. A., Herweh C., Bendszus M., Hoffmann J., Kargus S. Interdisciplinary treatment algorithm for facial high-flow arteriovenous malformations, and review of the literature. Journal of Cranio-Maxillo-Facial Surgery 46 (2018) 765-772.

[18] Fernandez AL. Surgical treatment of vascular malformations. An Sist Sanit Navar 2004; 27 (Suppl 1): 127-132.

[19] Riles TS, Berenstein A, Fisher FS, Persky MS, Madrid M. Reconstruction of the ligated external carotid artery for embolisation of cervicofacial arteriovenous malformations. Journal of vascular surgery 1993; 17 (3): 491-498.

[20] Chen W, Ye J, Xu L, Huang Z, Zhang D. A mutidisciplinary approach to treating maxillofacial arteriovenous malformation in children. Oral surg oral med oral pathol oral radiol endod 2009; 108: 41-47.

[21] White RI, Pollak J, Persing J, Henderson KJ, Thomson JG, Burdge CM. Long term outcome of embolotherapy and surgery for high-flow extremity arteriovenous malformations. JVIR 2000; 11: 1285-1295.

[22] Su L., Wang D., Han Y., Wang Z., Zheng L., Fan X. Absolute Ethanol Embolization of Infiltrating-diffuse Extracranial Arteriovenous Malformations in the Head and Neck. Eur J Vasc Endovasc Surg (2015) 50, 114-121.

[23] Mulliken JB. Cutaneous vascular anomalies. Semin Vasc Surg 1993; 6: 204-18.

[24] Lee BB, Do YS, Byun HS, Choo IW, Kim DI, Huk SH. Advanced management of venous malformation (VM) with ethanol sclerotherapy: mid-term results. J Vasc Surg 2003; 37: 533-8.

[25] Juszkat R, Zabicki B, Checinski P, Gabriel M, Matar N. Endovascular treatment of arteriovenous malformation. Aesth Plast Surg 2009; 33: 639-642.

[26] Kim S. H., Han S. H., Song Y., Park C. S., Song J. J. Arteriovenous malformation of the external ear: a clinical assessment with a scoping review of the literature. Braz J Otorhinolaryngol. 2017; 83 (6): 683-690. 